OPEN ACCESS

Citation: Hincapié C, Ascuntar J, León A, Jaimes F. Communityacquired pneumonia: comparison of three mortality prediction scores in the emergency department. Colomb Méd (Cali), 2021; 52(4):e2044287

http://doi.org/10.25100/ cm.v52i 4.4287

Received: 14 Apr 2020

Revised: 30 Mar 2021

Accepted : 25 Aug 2021

Published: 23 Oct 2021

Keywords:

Sepsis, pneumonia, clinical decision rules, mortality.

Palabras clave:

Sepsis, neumonía, reglas de decisión clínica, mortalidad

Copyright: () 2021 Universidad del Valle

(c) (1) $(-)$

ORIGINAL ARTICLE

\title{
Validation and comparison of three mortality prediction scores in emergency department patients with community-acquired pneumonia
}

\section{Validación y comparación de tres puntuaciones de predicción de mortalidad por neumonía adquirida en la comunidad, en pacientes del servicio de urgencias}

\author{
Carolina Hincapié ${ }^{1}$ (D) Johana Ascuntar ${ }^{1}$ (D) Alba León ${ }^{1}$ (D) Fabián Jaimes ${ }^{1,2}$ \\ fabian.jaimes@udea.edu.co \\ 1 Universidad de Antioquia; GRAEPIC - Clinical Epidemiology Academic Group (Grupo Académico de \\ Epidemiología Clínica); Medellín, Colombia. 2 Universidad de Antioquia, School of Medicine, Department \\ of Internal Medicine, Medellín, Colombia
}

\section{Abstract}

\section{Background:}

qSOFA is a score to identify patients with suspected infection and risk of complications. Its criteria are like those evaluated in prognostic scores for pneumonia (CRB-65 - CURB$65)$, but it is not clear which is best for predicting mortality and admission to the ICU.

\section{Objective:}

Compare three scores (CURB-65, CRB-65 and qSOFA) to determine the best tool to identify emergency department patients with pneumonia at increased risk of mortality or intensive care unit (ICU) admission.

\section{Methods:}

Secondary analysis of three prospective cohorts of patients hospitalized with diagnosis of pneumonia in five Colombian hospitals. Validation and comparison of the score's accuracies were performed by means of discrimination and calibration measures.

\section{Results:}

Cohorts 1, 2 and 3 included 158, 745 and 207 patients, with mortality rates of 32.3\%, $17.2 \%$ and $18.4 \%$, and admission to ICU was required for $52.5 \%, 43.5 \%$ and $25.6 \%$, respectively. The best AUC-ROC for mortality was for CURB-65 in cohort 3 (AUC$\mathrm{ROC}=0.67)$. The calibration was adequate $(p>0.05)$ for the three scores.

\section{Conclusions:}

None of these scores proved to be an appropriate predictor for mortality and admission to the ICU. Furthermore, the CRB 65 exhibited the lowest discriminative ability. 
Conflicts of interest:

The authors declare that they have no known competing financial interests or personal relationships that could have appeared to influence the work reported in this paper.

Acknowledgements:

We thank to Dr. Juan Jose Hurtado, Dr. Hernan Aguirre, and Dra. Ines Pachón for their support.

Funding:

Supported by COLCIENCIAS -

Administrative Department of Science, Technology, and Innovation (grants codes 111556933362, 111556933448, 111556933334) and the Universidad de Antioquia

Corresponding author:

Fabián Jaimes. E-mail address:fabian. jaimes@udea.edu.co Telephone: [57+4] 219 2432. Fax: [57+4] 2638282. Direction postal: $050010 \mathrm{E}$

\section{Resumen}

\section{Introducción:}

El qSOFA es un nuevo puntaje propuesto para ayudar a identificar pacientes con sospecha de infección y con alta probabilidad de desarrollar complicaciones graves. Los criterios del qSOFA son similares a los evaluados en los puntajes de pronóstico usados tradicionalmente en neumonía (CRB-65 y CURB-65), pero no está claro cuál es mejor para predecir la mortalidad y la admisión a la $\mathrm{UCl}$ en pacientes con neumonía en el servicio de urgencias

\section{Objetivo:}

Comparar tres puntajes (CURB-65, CRB-65 y qSOFA) para determinar la mejor herramienta para identificar en servicios de urgencias a los pacientes con neumonía con mayor riesgo de mortalidad o ingreso en la unidad de cuidados intensivos ( $\mathrm{UCl}$ ).

\section{Métodos:}

Análisis secundario de datos de tres estudios de cohorte prospectivos con pacientes atendidos por urgencias con diagnóstico de neumonía en 5 hospitales de Colombia. Se realizó validación y comparación de la exactitud de los puntajes por medio de medidas de discriminación y de calibración.

\section{Resultados:}

Las cohortes 1, 2 y 3 incluyeron 158, 745 y 207 pacientes, con mortalidad de 32.3\%, $17.2 \%$ y $18.4 \%$, respectivamente. Se requirió la admisión a la UCI para $52.5 \%, 43.5 \%$ y $25.6 \%$ pacientes3, respectivamente. La mejor AUC-ROC para mortalidad fue para CURB-65 en la cohorte 3 (AUC-ROC= 0.67). La calibración de los modelos fue adecuada para los tres puntajes $(P>0.05)$.

\section{Conclusiones:}

Ninguno de estos puntajes demostró ser un predictor adecuado de mortalidad e ingreso en UCl. Además, el CRB 65 mostró la capacidad discriminativa más baja. 


\section{Remark}

\section{1) Why was this study conducted?}

The CURB-65, CRB-65 and qSOFA were designed to identify patients at increased risk of complications and mortality. These scores share clinical variables in their compositions and community-acquired pneumonia is the main cause of sepsis; therefore, exploring potential differences in their performance as prognosis models would have implications for clinical practice.

\section{2) What were the most relevant results of the study?}

We did not find either the qSOFA, CURB-65 or CRB-65 to be adequate tools for discriminating hospital mortality or ICU admission in three cohorts of patients with community-acquired pneumonia, who were admitted to emergency departments in 5 reference hospitals in Medellín, Colombia.

\section{3) What do these results contribute?}

The qSOFA, CURB-65 and CRB-65 were all found to be ineffective predictive tools for mortality and admission to the ICU in our cohorts, therefore it is necessary to develop and validate predictive models of prognosis of community-acquired pneumonia that are useful for the Colombian population.

\section{Introduction}

Pneumonia is a significant cause of sepsis worldwide, representing approximately half of all cases, and is the second most frequent cause of sepsis in Colombia ${ }^{1,2}$. Globally, pneumonia confers a high risk of mortality ${ }^{3,4}$. Between 2005 and 2012 in Colombia, acute respiratory infection was the number one cause of death from communicable diseases, with $48.6 \%$ of the cases, representing $56.2 \%$ of deaths from communicable diseases in women and $43.1 \%$ in men ${ }^{5}$.

Providing health care to patients with severe infections carries high cost to a state and its health system. These infections have a challenging clinical approach because they do not have simple and specific prognostic markers that allow early identification of individuals at risk who warrant differential care. Therefore, it is important to have useful clinical tools to estimate the risk of death or complications in emergency department patients with suspected infections. Several studies have been conducted to define a mortality predictive score specifically for pneumonia, and the CURB-65 and CRB-65 scores have been widely used due to their easy application, compared with other ones such as the PSI (Pneumonia Severity Index $)^{6}$. Recently, the third consensus in sepsis (SEPSIS 3) encouraged the implementation of qSOFA (quick sepsis-related organ failure assessment) score in adult patients suspected of having an acute bacterial infection for early identification of those on worse prognosis ${ }^{7}$. The Colombian Ministry of Health ${ }^{8}$, as well as the Argentine Society of Infectious Diseases ${ }^{9}$, and the Mexican Institute of Social Security ${ }^{10}$ in their guidelines for the management of patients with community-acquired pneumonia, recommend implementing CURB-65, despite the lack of local studies to confirm and validate this recommendation ${ }^{7}$.

The CURB-65, CRB-65 and qSOFA were designed to identify patients at increased risk of complications and mortality. These scores share clinical variables in their compositions and community-acquired pneumonia is the main cause of sepsis; therefore, exploring potential differences in their performance as prognosis models would have implications for clinical 
practice. Likewise, it is necessary to validate any multivariable model that has been developed for prognostic or diagnostic purposes for a clinical issue in independent populations ${ }^{11}$. Therefore, this study aimed to validate and compare the three scores to determine the best tool to identify emergency department patients with pneumonia who are at increased risk of mortality or intensive care unit (ICU) admission.

\section{Materials and Methods}

\section{Study design and setting}

This analysis was performed using three prospective cohort studies developed between 2013 and 2016 in five emergency departments of the city of Medellín: Hospital Universitario San Vicente Fundación (560 adult inpatient beds and 45 ICU beds in 4 units), the University Health Services Provider Institution IPS Universitaria Clinica León XIII (450 adult inpatient beds and 24 ICU beds in 2 units), Hospital Pablo Tobón Uribe (360 adult inpatient beds and 40 UCI beds in 3 units), Hospital General de Medellín (442 beds) and Clínica Las Américas (304 beds) ${ }^{12-14}$. The first cohort was recruited from the emergency department of three tertiary care hospitals: Hospital Pablo Tobón Uribe, Hospital General de Medellín and Clínica Las Américas (2013-2016). The second was from the emergency departments of three tertiary care hospitals, Hospital Universitario San Vicente Fundación, Institución Universitaria Clínica León XIII and Hospital Pablo Tobón Uribe (2014-2016), and the third was from the emergency service of the Hospital Universitario San Vicente Fundación (2014-2016).

\section{Source of data}

For each of the original cohorts, trained research assistants collected data based on electronic medical records in a systematic way, reviewing all admissions to hospital institutions and screening all patients admitted for emergencies with a diagnosis of infection, sepsis, severe sepsis or shock. The definition of the infection source and the presence of organ dysfunction or septic shock were verified with the data extracted from the medical history records in the first 6 hours. To assess the data accuracy, the information was evaluated periodically by the coinvestigators. This information was recorded using forms designed specifically for each of the investigations and then stored in electronic databases. Given that the cohorts were prospective, the evaluation of predictors was independent from knowledge of the outcomes of interest.

Additionally, it was necessary for this study to recover the BUN (blood urea nitrogen) value upon hospital admission for patients at Hospital Universitario San Vicente Fundación and Hospital Pablo Tobón Uribe. The data collection process took information confidentiality into account and was approved by the ethics committees of each of the participating institutions.

\section{Participants}

For the current study, the inclusion criteria were patients who had entered the previous studies with a diagnosis of pneumonia. For cohort 1, the Centers for Disease Control and Prevention (CDC) criteria for infection were used for inclusion, cohort 2 consisted of suspected infection with at least one organ dysfunction criterion, and cohort 3 consisted of clinical suspicion of infection. As common criteria exclusion, we found for the 3 cohorts: patients that were early discharge or referenced to another institution, and patients with do-not-resuscitate orders or terminal diseases (Annex 1). No additional exclusion criteria that had not been considered in the original studies were used in the present study (12-14).

\section{Variables}

The primary outcome was hospital mortality; ICU admission was included as a secondary outcome. 


\section{The predictor variables:}

qSOFA: This severity prediction score includes scoring variables on the Glasgow Coma Scale $\leq 14$, systolic blood pressure $\leq 100 \mathrm{mmHg}$, and respiratory rate $\geq 22$ breaths per minute, with one point for each variable, for a total score between 0 and 3 . It is proposed that the presence of two of these three criteria could predict mortality in patients with suspected infection outside the ICU (7).

CURB-65: This score includes the variables confusion (Glasgow score $<15$ ), urea $>7 \mathrm{mmol} / \mathrm{L}$, respiratory rate $\geq 30$, systolic pressure $<90$ or diastolic $<60 \mathrm{mmHg}$, age $\geq 65$ years, with one point for each variable, for a total score between 0 and 5 . It is proposed that the presence of three or more points could predict mortality in patients with community-acquired pneumonia ${ }^{15}$.

CRB-65: This score includes the variables described above in the CURB-65 excepting the urea information. The score includes one point for each variable, for a total score between 0 and 4 . It is proposed that the presence of three or more points could predict mortality in communityacquired pneumonia patients ${ }^{15}$.

\section{Sample size}

Given that this was a secondary analysis of data, there was no calculation of sample size because the analysis was performed with patients of the respective cohorts that met the inclusion criteria. However, the power for the expected difference in the areas under the curve was calculated from a fixed number of patients and considered a type I error fixed at 0.05 . The calculation was based on the formula described by Hanley and McNeil ${ }^{16,17}$.

With a fixed sample size of 158, 745 and 207 patients for cohorts 1, 2 and 3, respectively, an alpha of 0.05 and taking the observed values of the AUC-ROC (area under the ROC curve) as $\theta 1$ : 0.7 and $\theta 2$ : 0.77 (based on the study by Kolditz et al. ${ }^{18}$ because we lacked this information locally), we found an estimation of power of $0.52,0.98$ and 0.62 , respectively.

\section{Statistical methods}

The quantitative variables with a normal distribution are presented as means and standard deviations, while those without a normal distribution are expressed as medians and interquartile ranges (IQRs).

A validation and comparison of the three predictive models (CURB-65, CRB-65 and qSOFA) was performed in terms of prognosis. To determine the accuracy of the prediction of the models, it was necessary to examine both the calibration and the discrimination. Calibration compares and establishes the agreement between observed and expected events, while discrimination establishes the ability with which the score distinguishes between individuals who experience or do not experience the event of interest ${ }^{19}$. The performance of the scores in terms of discrimination was determined based on the area under the receiver operating characteristics curve (AUC-ROC) based on the models defined as the sum of the corresponding predictors. The differences between the AUC-ROC were tested using the DeLong-DeLong statistic ${ }^{20}$. The calibration was determined by the degree of correspondence given by the Hosmer-Lemeshow goodness-of-fit test $(\mathrm{p}>0.05)$. Additionally, calibration curves were performed based on the results of the models in each of the cohorts.

The operative characteristics for prediction of mortality and ICU need for each of the scores were then estimated, taking two or more points for the qSOFA and 3 or more for both the CURB-65 and CRB-65, based on the original proposal of the models indicating these cutoff points as high risk of mortality. Likewise, the performance of each of the predictive models was analyzed according to all possible cutoff points and compared with the originally proposed cohort points. To calculate the sensitivity, specificity, predictive values and the likelihood ratios of the mentioned scores with their respective cutoff points, Bayes theorem was used, considering mortality and ICU need as a reference test or gold standard. 
In the main analysis, missing data were considered as abnormal values (worst-case scenario). Additionally, a sensitivity analysis was performed with two additional models: the best scenario, considering the missing data as normal values, and with a multivariate normal regression (MVN), multiple imputation technique, taking the BUN, age, gender, Charlson index, SOFA and Acute Physiology, Age, Chronic Health Evaluation II (APACHEII) as independent values.

Statistical analyses were performed with the Stata $14^{\circ}$ software. The results are presented with their respective $95 \%$ confidence intervals (CI), and a significance level of $\mathrm{p}<0.05$ was applied. Publication standards given by the transparent reporting of a multivariable prediction model for individual prognosis or diagnosis (TRIPOD) guidelines were followed ${ }^{11}$.

\section{Results}

A total of 158, 745 and 207 patients were analyzed for cohorts 1, 2 and 3, respectively. In the same order, the median age was $70(\mathrm{IQR}=56-81), 66(\mathrm{IQR}=54-77)$ and $60(\mathrm{IQR}=44-75)$ years; $34.2 \%, 48.9 \%$ and $44.4 \%$ were female; $52.5 \%, 43.5 \%$ and $25.6 \%$ required admission to the ICU; and $32.3 \%, 17.2 \%$ and $18.4 \%$ died during hospitalization (Table 1). Blood cultures were requested in $95.6 \%, 84.8 \%$ and $84.5 \%$ and germs were isolated for $23.2 \%, 10.8 \%$ and $9.1 \%$ of the patients in cohorts 1,2, and 3, respectively. The most frequent microorganisms found in each of the cohorts were Streptococcus pneumoniae, Klebsiella pneumoniae, Haemophilus influenzae and Escherichia coli (Table 2).

Abbreviations: SOFA, Sequential Organ Failure Assessment; APACHE II, Acute Physiology and Chronic Health Evaluation II; RR, respiratory rate; SAP, systolic arterial pressure; DAP, diastolic arterial pressure; MAP, mean arterial pressure; BUN, blood urea nitrogen. The quantitative variables were expressed as the medians and their respective interquartile range; categorical variables are shown in absolute and relative frequencies.

Table 1. Baseline Characteristics of the study population, according to the original cohorts.

\begin{tabular}{|c|c|c|c|}
\hline & Cohort $1(n=158)$ & Cohort $2(n=745)$ & Cohort $3(n=207)$ \\
\hline \multicolumn{4}{|l|}{ Characteristics } \\
\hline Age, years & $70(56-81)$ & $66(54-77)$ & $60(44-75)$ \\
\hline Female sex & $54(34.2 \%)$ & $364(48.9 \%)$ & $92(44.4 \%)$ \\
\hline CDC criteria & $158(100 \%)$ & $628(84.75 \%)$ & $187(90.34 \%)$ \\
\hline \multicolumn{4}{|l|}{ Severity } \\
\hline Charlson index & $1(0-3)$ & $1(0-2)$ & $1(0-2)$ \\
\hline SOFA & $4(3-6)$ & $4(3-6)$ & $3(2-5)$ \\
\hline APACHE II & $17(12-21)$ & $15(11-19)$ & $13(9-17)$ \\
\hline \multicolumn{4}{|l|}{ Variables } \\
\hline $\mathrm{RR}$ & $24(20-28)$ & $22(19-27)$ & $23(19-26)$ \\
\hline SAP & $110(90-130)$ & $113(92-132)$ & $120(100-140)$ \\
\hline DAP & $60(49-72)$ & $68(55-80)$ & $76(60-84)$ \\
\hline MAP & $76(64-90)$ & $83(68-97)$ & $91(73-101)$ \\
\hline Glasgow coma scale & $15(14-15)$ & $15(15-15)$ & $15(15-15)$ \\
\hline BUN & $\mathrm{n}=14327.1(16.3-45.2)$ & $\mathrm{n}=70421.5(14-33.4)$ & $\mathrm{n}=20618(13-29)$ \\
\hline$\geq 65$ years & $93(58.9 \%)$ & $393(52.8 \%)$ & $89(43 \%)$ \\
\hline \multicolumn{4}{|l|}{ Scores } \\
\hline qSOFA & $1(1-2)$ & $1(1-2)$ & $1(0-1)$ \\
\hline CURB 65 & $2(2-3)$ & $2(1-3)$ & $1(1-2)$ \\
\hline CRB 65 & $2(1-2)$ & $1(1-2)$ & $1(0-2)$ \\
\hline \multicolumn{4}{|l|}{ Outcomes } \\
\hline ICU & $83(52.5 \%)$ & $324(43.5 \%)$ & $53(25.6 \%)$ \\
\hline Death & $51(32.3 \%)$ & $128(17.2 \%)$ & $38(18.4 \%)$ \\
\hline
\end{tabular}

Abbreviations: SOFA, Sequential Organ Failure Assessment; APACHE II, Acute Physiology and Chronic Health Evaluation II; RR, respiratory rate; SAP, systolic arterial pressure; DAP, diastolic arterial pressure; MAP, mean arterial pressure; BUN, blood urea nitrogen. The quantitative variables were expressed as the medians and their respective interquartile range; categorical variables are shown in absolute and relative frequencies. 
Table 2. Blood cultures and microbiological results in the study population, according to the original cohorts

\begin{tabular}{lrrr}
\hline & Cohort 1 $(\mathrm{n}=158)$ & Cohort 2(n=745) & Cohort 3 (n=207) \\
\hline Characteristics & & & \\
Blood culture requested & $151(95.6 \%)$ & $632(84.8 \%)$ & $175(84.5 \%)$ \\
Positive blood culture & $35(23.2 \%)$ & $68(10.8 \%)$ & $16(9.1 \%)$ \\
Main microorganisms & & & \\
Streptococcus pneumoniae & $13(8.6 \%)$ & $23(3.6 \%)$ & $6(3.4 \%)$ \\
Klebsiella pneumoniae & $6(4 \%)$ & $6(1 \%)$ & $1(1 \%)$ \\
Haemophilus influenza & $2(1.3 \%)$ & $8(1 \%)$ & $2(1.1 \%)$ \\
Staphylococcus aureus & $4(2.7 \%)$ & $9(1.4 \%)$ & $1 \%)$ \\
Escherichia coli & $5(3.3 \%)$ & $3(1 \%)$ & 0 \\
Pseudomonas aeruginosa & $2(1.3 \%)$ & $1 \%)$ & \\
\hline
\end{tabular}

For the outcome of admission to the ICU, discrimination was low for the three scores in the three cohorts. From the DeLong-DeLong statistic, a statistically significant difference was found between the AUC-ROC in cohorts 1 and 2 ( $\mathrm{P}<0.05)$ (Figure 1), with an AUC-ROC of 0.59 for the qSOFA, 0.43 for the CURB-65 and 0.44 for the CRB-65 in cohort 1 . For the mortality outcome, discrimination was not adequate in any of the three scores in any of the three cohorts. From the DeLong-DeLong statistic, a statistically significant difference was found in the AUC-ROC of cohorts 1 and 2 (Figure 2), with an AUC-ROC of 0.66 (95\% CI = 0.62- 0.71) for the CURB-65, $0.60(95 \% \mathrm{CI}=0.56-0.65)$ for the qSOFA and $0.63(95 \% \mathrm{CI}=$ $0.59-0.68)$ for the CRB -65 in cohort 2.

The calibration of the models was adequate in the study population for admission to the ICU and the mortality outcome, according to the Hosmer-Lemeshow statistic of the three scores in each of the cohorts ( $\mathrm{p}>0.05)$ (Table S1).

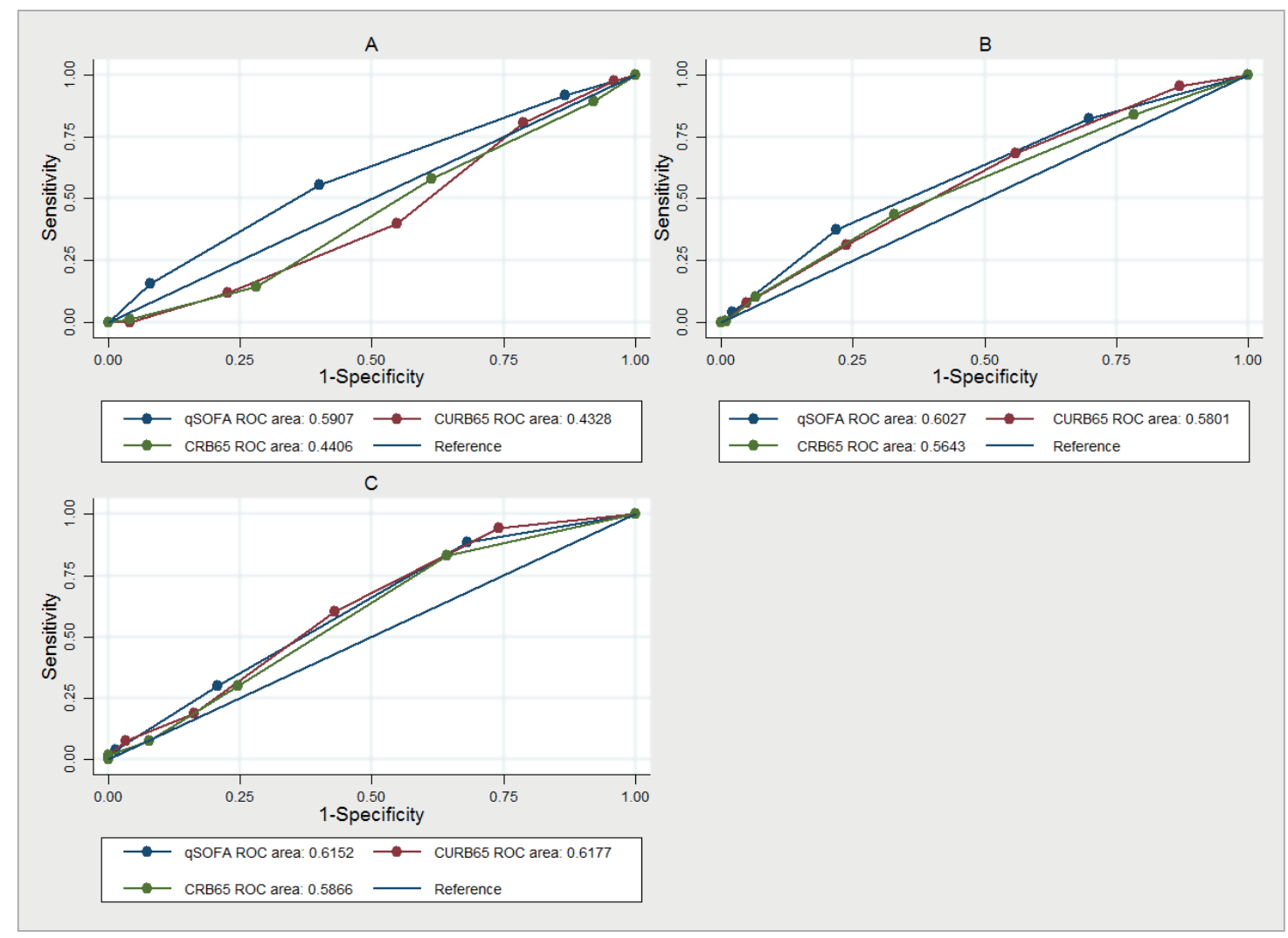

Figure 1. Receiver operating characteristic curve in the different cohorts for qSOFA, CURB-65 and CRB-65 in the discrimination of ICU admission. A. Cohort: 1- DeLong-DeLong $\mathrm{p}=0.0008$. B. Cohort 2- DeLong-DeLong $\mathrm{p}=0.0402$. C. Cohort 3 - DeLong-DeLong $\mathrm{p}=0.3403$. 


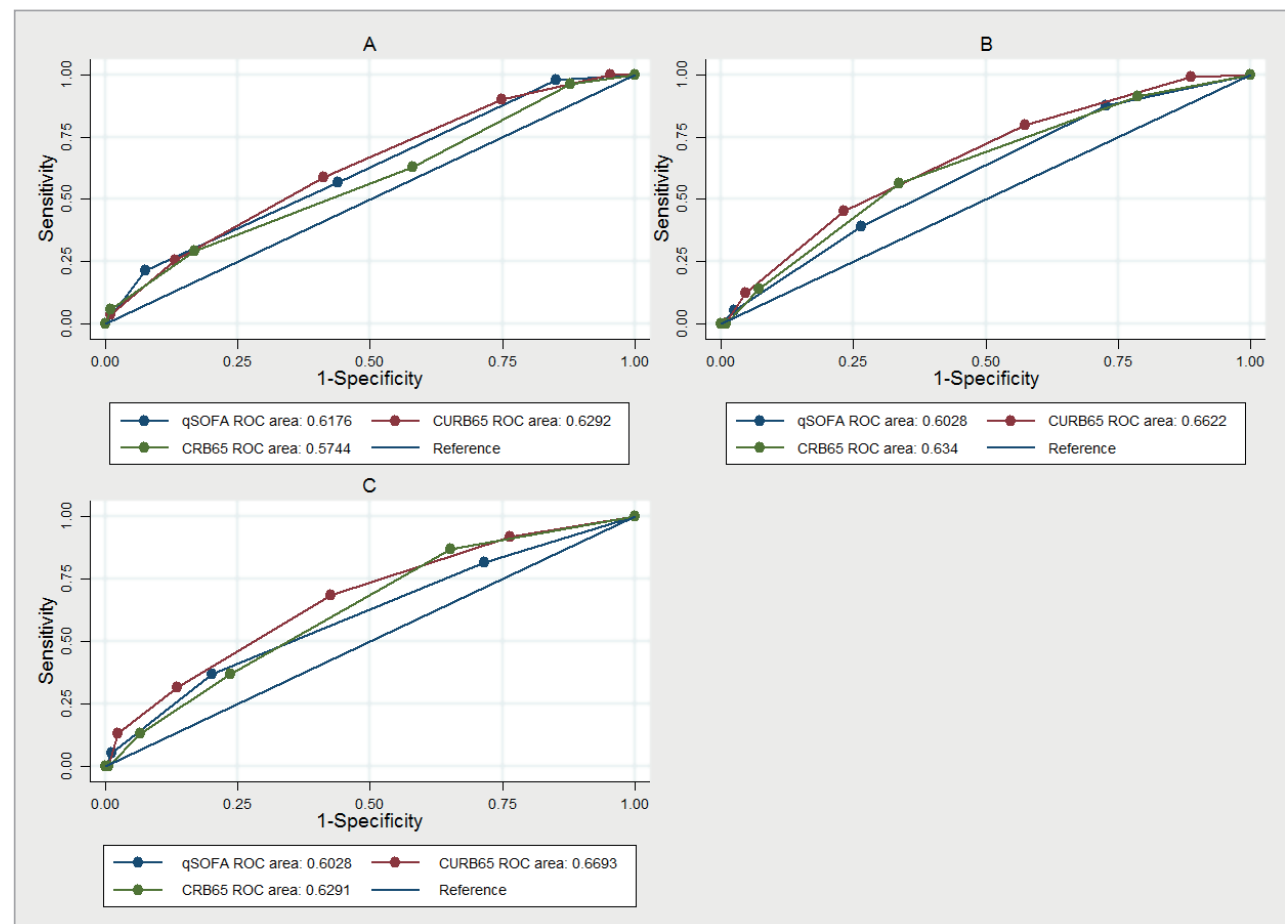

Figure 2. Receiver operating characteristic curve in the different cohorts for qSOFA, CURB-65 and CRB-65 in the discrimination of mortality. A. Cohort 1 (DeLong-DeLong $\mathrm{p}=0.0108$ ). B. Cohort 2 (DeLong-DeLong $\mathrm{p}=0.0218$ ). C. Cohort (DeLong-DeLong $\mathrm{p}=0.1606$ ).

Additionally, calibration curves were performed for both outcomes in the different models in each of the cohorts, and a high degree of correspondence of the scores was shown in most of the cohorts (Supplementary Figure S1 and andS2).S2).

Regarding the performance of the models in their operative characteristics, the greatest sensitivity for ICU need was with the qSOFA (55.4\%) and for mortality was with CURB-65 $(58.8 \%)$ in cohort 1 . The greatest specificity was with CRB-65 for both ICU need and mortality, with $93.5 \%$ and $93.4 \%$ in cohorts 2 and 3 , respectively. The lowest performance in predicting mortality in terms of sensitivity was for the CRB-65 in cohort 3 (13.2\%), for specificity it was for the qSOFA in cohort 1 (43.9\%) and for the positive predictive value it was the CRB-65 in cohort 3 (Tables S2and andS3).S3).

\section{Discussion}

We found that qSOFA, CURB-65 or CRB-65 were not optimal in discriminating hospital mortality or ICU admission in three cohorts of patients with community-acquired pneumonia admitted to five hospitals in Medellín. However, looking at the AUC, sensitivity and negative predictive value values, CURB-65 appeared to consistently perform better than the other two tools with respect to mortality discrimination. In contrast, with regard to calibration, it was possible to demonstrate a good performance for the three scores in the 3 cohorts. Nevertheless, a lack of good discriminative performance indicates that these scoring systems should not be used as predictive tools ${ }^{19,21}$.

It is necessary to account for the setting of the studies that originally developed the scores: the CURB-65 and the CRB-65 were developed in the United Kingdom, New Zealand and the Netherlands ${ }^{22}$ more than 20 years ago, countries with a community-acquired pneumonia associated mortality lower than in Colombia ( $9 \%$ vs $17-32 \%)$. On the other hand, the qSOFA was derived from a very recent cohort ${ }^{23}$ that used a clinical spectrum beyond pneumonia and showed a hospital mortality of only $4 \%$. In 2006, Capelastegui et al. (, ${ }^{24}$, showed a similar 
performance between the CURB- 65 and CRB-65 scores for mortality at 30 days with an AUC over 0.85 . Subsequently, Man et al. compared these prediction rules for 30-day mortality in patients with community-acquired pneumonia and found AUCs higher than the ones observed in the present study ${ }^{25}$.

In the original studies that served as the basis for the development of qSOFA, Seymour et al. found a good performance for the prediction of in-hospital mortality ${ }^{26}$. Subsequently, Wang et al. performed a secondary analysis of data from a prospective cohort where they evaluated the performance of qSOFA in patients with an infection diagnosis who admitted to the emergency department, and found the score did not have a good performance (AUC $=0.66$ ) for 28 -day mortality ${ }^{27}$.

Previous studies have demonstrated these scores underestimated risk in patients with community acquired pneumonia. A couple of years ago, Chen et al. compared the performance of the qSOFA, CRB and CRB-65 with respect to mortality and admission to the ICU. The AUC-ROC values of the qSOFA for the prediction of mortality at 28 days were similar to those of the and CRB- 65 scores, 0.655 vs 0.661 respectively. Likewise, the prediction of admission to the ICU showed similar discrimination measures, 0.666 vs 0.685 respectively ${ }^{28}$. These results are consistent with ours in terms of discrimination, both for admission to the ICU and for mortality, despite its large sample size and being performed in a single hospital, which could result in less variability in the overall sample. In Germany, Kolditz et al. ${ }^{18}$, compared qSOFA with the CRB and CRB-65 for 30-day mortality in patients with community-acquired pneumonia. They found that the AUC-ROC results favored the CRB-65 compared to the qSOFA, AUC-ROC 0.77 vs 0.70 respectively.

More recently, three different studies ${ }^{29-31}$ show the comparison of severity scores in patients with COVID-19 pneumonia, all of them shows that that CURB-65 could be better compared with qSOFA at estimating mortality. Guohui et al. ${ }^{30}$, found an AUC for discharge mortalitity of 0.85 for CURB-65, 0.80 for CRB-65 and 0.73 for and qSOFA. Bradley et al. found an AUC for 30 days mortalitity of 0.75 for CURB- 65 and 0.62 for and qSOFA. Lazar Neto et al. found an AUC for 30 days in hospital mortalitity of 0.74 for CURB- 65 and 0.63 for and qSOFA.

As shown in the studies presented previously, the performance of the scores changed significantly among all cohorts due to their differences, include the distribution of etiological agents, coexisting diseases, social support, availability of resources and medical behaviors, including the ICU admission criteria. In our study, these scores performance varied even though the cohorts were from the same city, which can be explained by the variability in the patient inclusion criteria.

The AUC-ROC is a statistical parameter that allows the comparison of predictive models of diagnosis or prognosis in terms of discrimination capacity, and it is reasonable to use an AUCROC $>0.75$ as a reference of acceptable performance. However, this statistical measure does not allow a direct clinical interpretation, and this limitation in predictive models is a constant in the literature on this topic, for this reason it is always necessary to evaluate simultaneously their operative characteristics. Regarding calibration, none of the mentioned studies above accounted for this in the statistical analysis. The critical importance of poor calibration is often underestimated. This can lead to a decrease in clinical utility; the implementation of a predictive tool with poor calibration could even lead to making decisions that are harmful to the patient ${ }^{32}$. Future studies could consider other variables for score calculations, such as variables related to the microbiological agent, pulse oximetry, temperature, and comorbidities such as chronic obstructive pulmonary disease, congestive heart failure, and immunosuppression, among others.

One of the limitations of our study was the sample size. We based the difference of 0.7-0.77 between the discrimination (AUC-ROC) of CRB-65 and qSOFA scores on partial information from Kolditz et al ${ }^{18}$. This difference, however, does not necessarily have a clinical basis and did not consider that all scores had a final poor discrimination performance (AUC $<0.75$ ). The 
traditional approximation of the sample size calculation in predictive models defines a value of at least 10 outcomes for each independent variable ${ }^{33,34}$. For comparisons between models, exclusively by means of discrimination, we based the sample size formula on the AUC-ROC comparison by Hanley-McNeil ${ }^{16,17}$. However, specifically for the validation of predictive models, there is no clear indication of the sample size calculation, and although some authors have suggested a minimum of 100 outcomes, many studies do not consider this aspect ${ }^{35,36}$. On the other hand, the collection was performed in 5 institutions that are recognized as high quality health care centers, which can lead to a selection bias. However, the three cohorts had different inclusion criteria, which significantly improved the clinical spectrum of the study population. Another limitation was that despite being prospectively constructed cohorts, this study provides a secondary analysis of data, giving rise to missing urea values for some participants. These missing data were considered as abnormal values, which could generate a differential or nondifferential classification bias. The missing data represented only $5 \%$, however, and the sensitivity analysis with different scenarios did not improve the performance of the models.

A predictive model is not of practical use if it cannot discriminate and be calibrated at the same time: to properly separate those who present the condition from those who do not, is as important as whether there is agreement between observed and expected events ${ }^{21}$. Unlike the supervision required for new medical technologies, prediction systems are not subjected to strict judgments, despite the potential risk of affecting a greater number of patients due to their extensive implementation.

\section{Conclusion}

In the three independent cohorts of patients admitted by the emergency department with pneumonia, the qSOFA, CURB-65 and CRB- 65 were all found to be limited predictive tools for mortality and admission to the ICU. Furthermore, the CRB-65 exhibited the lowest discriminative ability.

\section{References}

1. Rodriguez F, Barrera L, De La Rosa G, Dennis R, Duenas C, Granados M, et al. The epidemiology of sepsis in Colombia: a prospective multicenter cohort study in ten university hospitals. Crit Care Med. 2011;39(7):167582. Doi: 10.1097/CCM.0b013e318218a35e.

2. Angus DC, van der Poll T. Severe sepsis and septic shock. New Engl J Med. 2013; 369(9): 840-51. Doi: 10.1056/NEJMra1208623.

3. Lindenauer PK, Lagu T, Shieh MS, Pekow PS, Rothberg MB. Association of diagnostic coding with trends in hospitalizations and mortality of patients with pneumonia, 2003-2009. JAMA. 2012; 307(13): 1405-13. Doi: 10.1001/jama.2012.384.

4. Mandell LA, Wunderink RG, Anzueto A, Bartlett JG, Campbell GD, Dean NC, et al. Infectious Diseases Society of America/American Thoracic Society consensus guidelines on the management of communityacquired pneumonia in adults. Clin Infect Dis. 2007; 44 Suppl 2: S27-72. Doi: 10.1086/511159.

5. Ministerio de Salud y Protección Social. Analisis de Situación de salud de Colombia 2014. Bogota: Ministerio de Salud y Protección Social; 2014. Available in: https://www.minsalud.gov.co/sites/rid/Lists/BibliotecaDigital/ RIDE/VS/ED/PSP/ASIS_2014_v11.pdf.

6. Chalmers JD, Singanayagam A, Akram AR, Mandal P, Short PM, Choudhury G, et al. Severity assessment tools for predicting mortality in hospitalised patients with community-acquired pneumonia. Systematic review and meta-analysis. Thorax. 2010; 65(10): 878-83. Doi: 10.1136/thx.2009.133280.

7. Singer M, Deutschman CS, Seymour CW, Shankar-Hari M, Annane D, Bauer M, et al. The Third International Consensus Definitions for Sepsis and Septic Shock (Sepsis-3). JAMA. 2016; 315(8): 801-10. Doi: 10.1001/ jama.2016.0287. 
8. Ministerio de la Protección Social. Guías para Manejo de Urgencias. 3a Edición. Bogota: Ministerio de la Protección; 2009

9. Lopardo G, Basombrío A, Clara L, Desse J, De Vedia L, Libero E, et al. Neumonía adquirida de la comunidad en adultos: recomendaciones sobre su atención. Medicina (Buenos Aires). 2015;75:245-57.

10. Instituto Mexicano del Seguro Social. Prevención, Diagnóstico y Tratamiento de la Neunonía Adquirida en la Comunidad. Evidencias y Recomendaciones. Catálogo Maestro de Guías de Práctica Clínica: IMSS-234-09. México, DF:

11. Collins GS, Reitsma JB, Altman DG, Moons KG. Transparent reporting of a multivariable prediction model for individual prognosis or diagnosis (TRIPOD): the TRIPOD statement. BMJ. 2015; 350: g7594. Doi: 10.1136/ bmj.g7594.

12. Londono J, Nino C, Archila A, Valencia M, Cardenas D, Perdomo M, et al. Antibiotics has more impact on mortality than other early goal-directed therapy components in patients with sepsis: An instrumental variable analysis. J Crit Care. 2018;48:191-7. Doi: 10.1016/j.jcrc.2018.08.035.

13. Castano P, Plaza M, Molina F, Hincapie C, Maya W, Catano J, et al. Antimicrobial agent prescription: a prospective cohort study in patients with sepsis and septic shock. Trop Med Internat Health. 2019; 24(2): 17584. Doi: $10.1111 / \mathrm{tmi} .13186$.

14. Londono J, Nino C, Diaz J, Morales C, Leon J, Bernal E, et al. Association of clinical hypoperfusion variables with lactate clearance and hospital mortality. Shock. 2018; 50(3): 286-92. Doi: 10.1097/ SHK.0000000000001066.

15. Lim WS, van der Eerden MM, Laing R, Boersma WG, Karalus N, Town GI, et al. Defining community acquired pneumonia severity on presentation to hospital: an international derivation and validation study. Thorax. 2003;58(5):377. Doi: 10.1136/thorax.58.5.377.

16. Hanley JA, McNeil BJ. The meaning and use of the area under a receiver operating characteristic (ROC) curve. Radiology. 1982; 143(1): 29-36.17. Doi: 10.1148/radiology.143.1.7063747.

17. Hanley JA, McNeil BJ. A method of comparing the areas under receiver operating characteristic curves derived from the same cases. Radiology. 1983;148(3):839-43. Doi: 10.1148/radiology.148.3.6878708.

18. Kolditz M, Scherag A, Rohde G, Ewig S, Welte T, Pletz M. Comparison of the qSOFA and CRB-65 for risk prediction in patients with community-acquired pneumonia. Intensive Care Med. 2016;42(12):2108-10. Doi: 10.1007/s00134-016-4517-y.

19. Altman D, Royston P. What do we mean by validating a prognostic model? Stat Med. 2000;19(4):453 - 73. Doi: 10.1002/(SICI)1097-0258(20000229)19:4 \&It;453::AID-SIM350 \&gt;3.0.CO;2-5

20. DeLong ER, DeLong DM, Clarke-Pearson DL. Comparing the areas under two or more correlated receiver operating characteristic curves: a nonparametric approach. Biometrics. 1988; 44(3):837-45.

21. Cook NR. Statistical Evaluation of Prognostic versus Diagnostic Models: Beyond the ROC Curve. Clin Chem. 2008;54(1):17-23. Doi: 10.1373/clinchem.2007.096529

22. Lim WS, Macfarlane JT, Boswell TC, Harrison TG, Rose D, Leinonen M, et al. Study of community acquired pneumonia aetiology (SCAPA) in adults admitted to hospital: implications for management guidelines. Thorax. 2001;56(4):296-301. Doi: 10.1136/thorax.56.4.296.

23. Ranzani OT, Prina E, Menendez R, Ceccato A, Cilloniz C, Mendez R, et al. New Sepsis Definition (Sepsis-3) and Community-acquired Pneumonia Mortality. A Validation and Clinical Decision-Making Study. Am J Respirat Crit Care Med. 2017; 196(10): 1287-97. Doi: 10.1164/rccm.201611-2262OC. 
24. Capelastegui A, Espana PP, Quintana JM, Areitio I, Gorordo I, Egurrola M, et al. Validation of a predictive rule for the management of community-acquired pneumonia. Europ Respirat J. 2006;27(1):151-7. Doi:

10.1183/09031936.06.00062505.

25. Man SY, Lee N, Ip M, Antonio GE, Chau SS, Mak P, et al. Prospective comparison of three predictive rules for assessing severity of community-acquired pneumonia in Hong Kong. Thorax. 2007;62(4):348-53. Doi: 10.1136/thx.2006.069740

26. Seymour CW, Liu VX, Iwashyna TJ, Brunkhorst FM, Rea TD, Scherag A, et al. Assessment of Clinical Criteria for Sepsis: For the Third International Consensus Definitions for Sepsis and Septic Shock (Sepsis-3). JAMA. 2016;315(8):762-74. Doi: 10.1001/jama.2016.0288.

27. Wang JY, Chen YX, Guo SB, Mei X, Yang P. Predictive performance of quick Sepsis-related Organ Failure Assessment for mortality and ICU admission in patients with infection at the ED. Am J Emerg Med. 2016;34(9):1788-93. Doi: 10.1016/j.ajem.2016.06.0152

28. Chen YX, Wang JY, Guo SB. Use of CRB-65 and quick Sepsis-related Organ Failure Assessment to predict site of care and mortality in pneumonia patients in the emergency department: a retrospective study. Crit Care. 2016;20(1):167. Doi: 10.1186/s13054-016-1351-0.

29. Lazar Neto F, Marino LO, Torres A, Cilloniz C, Meirelles Marchini JF, Garcia de Alencar JC, et al. Communityacquired pneumonia severity assessment tools in patients hospitalized with COVID-19: a validation and clinical applicability study. Clin Microbiol Infect. 2021; 27(7) :1037.e1-1037.e8. Doi: 10.1016/j.cmi.2021.03.002.

30. Fan G, Tu C, Zhou F, Liu Z, Wang Y, Song B, et al. Comparison of severity scores for COVID-19 patients with pneumonia: a retrospective study. Eur Respir J. 2020;56(3): 2002113. Doi: 10.1183/13993003.02113-2020

31. Bradley P, Frost F, Tharmaratnam K, Wootton DG, Research NWCOfR. Utility of established prognostic scores in COVID-19 hospital admissions: multicentre prospective evaluation of CURB-65, NEWS2 and qSOFA. BMJ Open Respir Res. 2020;7(1):e000729. Doi: 10.1136/bmjresp-2020-000729.

32. Van Calster B, Vickers AJ. Calibration of risk prediction models: impact on decision-analytic performance. Medical Decision Making. 2015;35(2):162-9. Doi: 10.1177/0272989X14547233.

33. Vittinghoff E, McCulloch CE. Relaxing the rule of ten events per variable in logistic and Cox regression. Am J Epidemiol. 2007;165(6):710-8. Doi: 10.1093/aje/kwk052.

34. Courvoisier DS, Combescure C, Agoritsas T, Gayet-Ageron A, Perneger TV. Performance of logistic regression modeling: beyond the number of events per variable, the role of data structure. J Clin Epidemiol. 2011;64(9):993-1000. Doi: 10.1016/j.jclinepi.2010.11.012.

35. Vergouwe Y, Steyerberg EW, Eijkemans MJ, Habbema JD. Substantial effective sample sizes were required for external validation studies of predictive logistic regression models. J Clin Epidemiol. 2005;58(5):475-83. Doi: 10.1016/j.jclinepi.2004.06.017.

36. Steyerberg EW, Borsboom GJ, van Houwelingen HC, Eijkemans MJ, Habbema JD. Validation and updating of predictive logistic regression models: a study on sample size and shrinkage. Stat Med. 2004;23(16):2567-86. Doi: $10.1002 / \operatorname{sim} .1844$. 


\section{Annex 1. Inclusion and exclusion criteria.}

\section{Cohort 1}

Inclusion criteria: Patients with an infection in accordance with the clinical or microbiological criteria of the CDC definitions, and at least one organ/system dysfunction, based on the SOFA score $\geq 2$, produced or related to the infection, and detected within $24 \mathrm{~h}$ prior to being admitted to the study. For the HGM and CLA, in addition to the previous criteria, patients were required to have positive blood cultures. This condition was established because of problems in recruiting and the need to improve the efficiency in the analysis by the infectious disease specialists. Exclusion criteria: Patients referred to another hospital within the first $48 \mathrm{~h}$ of admission were excluded, as were patients with known infections who needed prolonged treatments (e.g. tuberculosis, nocardiosis, histoplasmosis) and patients with do-not-resuscitate orders or terminal diseases.

\section{Cohort 2}

Inclusion criteria: Patients $\geq 18$ years, admitted by the ER with a suspected or confirmed diagnosis of infection, sepsis, severe sepsis or septic shock; at least two criteria of systemic inflammatory-response-syndrome and systolic blood pressure $<90 \mathrm{mmHg}$ after a bolus of crystalloid of at least $20 \mathrm{ml} / \mathrm{kg}$, or a serum lactate $>4 \mathrm{mmol} / \mathrm{L}$.

Exclusion criteria: Refusal by the patient, family or attending physician to participate; pregnancy, myocardial infarction, stroke, asthmatic crisis, arrhythmia, trauma, gastrointestinal bleeding, seizure not due to meningitis, psychoactive substance overdose, surgery $<24$ hours, burns, CD 4 count $<50$ cells/mm3, hyperosmolar status, diabetic ketoacidosis or cirrhosis; released or referred in the first 24 hours, prior participation in the study, referral from another institution where the patient has been hospitalized $>24$ hours, or a Do-Not Resuscitate (DNR) order.

\section{Cohort 3}

Inclusion criteria: patients $\geq 18$ years admitted to the ER with an acute bacterial infection confirmed by clinical or laboratory evidence, in accordance with the CDC criteria; Furthermore, patients should be available to assess vital signs and for physical findings upon being admitted to the hospital.

Exclusion criteria: patients who were referred from another institution where they stayed for more than 24 hours; discharge or reference to another institution during the first 24 hours of admission; diseases that hinder the physical evaluation of clinical parameters, such as limb amputation, extensive burns or severe skin diseases, Raynaud's phenomenon or peripheral arterial disease; cirrhosis; mesenteric thrombosis; patient's refusal to participate; screening after 6 hours of being admitted to the ER; a previous participation in the study and a do not resuscitate order. 


\section{Tables}

Table S1. Calibration of the models for ICU admission and mortality

\begin{tabular}{|c|c|c|c|}
\hline & Cohort $1(n=158)$ & Cohort $2(n=745)$ & Cohort $3(n=207)$ \\
\hline \multicolumn{4}{|l|}{ ICU admission } \\
\hline Number of groups & 7 & 5 & 7 \\
\hline Hosmer-Lemeshow & 1.6 & 1.1 & 2.0 \\
\hline$p$-Value & 0.9002 & 0.7875 & 0.8449 \\
\hline \multicolumn{4}{|l|}{ CURB-65 } \\
\hline Number of groups & 10 & 10 & 9 \\
\hline Hosmer-Lemeshow & & 6.2 & 3.1 \\
\hline$p$-Value & 0.4355 & 0.6284 & 0.8749 \\
\hline \multicolumn{4}{|l|}{ CRB-65 } \\
\hline Number of groups & 10 & 8 & 7 \\
\hline Hosmer-Lemeshow & 12.1 & 3.9 & 3.3 \\
\hline$p$-Value & 0.1466 & 0.6941 & 0.6478 \\
\hline \multicolumn{4}{|l|}{$\begin{array}{l}\text { Mortality } \\
\text { qSOFA }\end{array}$} \\
\hline Number of groups & 6 & 6 & 4 \\
\hline Hosmer-Lemeshow & 4.6 & 1.1 & 0.14 \\
\hline$p$-Value & 0.3365 & 0.8985 & 0.9316 \\
\hline \multicolumn{4}{|l|}{ CURB-65 } \\
\hline Number of groups & 10 & 10 & 9 \\
\hline Hosmer-Lemeshow & 5 & 8.1 & 3.5 \\
\hline$p$-Value & 0.7612 & 0.4236 & 0.8309 \\
\hline \multicolumn{4}{|l|}{ CRB-65 } \\
\hline Number of groups & 10 & 7 & 7 \\
\hline Hosmer-Lemeshow & 5.7 & 6.42 & 1.9 \\
\hline$p$-Value & 0.6843 & 0.2672 & 0.8632 \\
\hline
\end{tabular}

Table S2. Operative characteristics for ICU admission prediction in qSOFA (with cut-off point $\geq 2$ ), CURB 65 (with cut-off point $\geq$ 3) and CRB 65 (with cut-off point $\geq 3$ ).

\begin{tabular}{|c|c|c|c|c|c|c|}
\hline & $\begin{array}{c}\text { Sensitivity } \\
(95 \% \text { CI })\end{array}$ & $\begin{array}{c}\text { Specificity } \\
(95 \% \text { CI })\end{array}$ & $\begin{array}{c}\text { PPV } \\
(95 \% \mathrm{CI})\end{array}$ & $\begin{array}{c}\text { NPV } \\
(95 \% \text { CI })\end{array}$ & $\mathrm{LR}+(\mathbf{9 5 \%} \mathrm{CI})$ & LR - $(95 \% \mathrm{CI})$ \\
\hline \multicolumn{7}{|l|}{ qSOFA } \\
\hline Cohort 1 & $55.4(44.1-66.7)$ & $60.0(48.3-71.8)$ & $60.5(48.9-72.2)$ & $54.9(43.5-66.3)$ & $1.4(1-1.9)$ & $0.7(0.6-1.0)$ \\
\hline Cohort 2 & $37.4(31.9-42.8)$ & $78.2(74.1-82.2)$ & $56.8(49.9-63.7)$ & $61.8(57.6-66.1)$ & $1.7(1.4-2.2)$ & $0.8(0.7-0.9)$ \\
\hline Cohort 3 & $30.2(16.9-43.5)$ & $79.2(72.5-86)$ & $33.3(19-47.7)$ & $76.7(69.9-83.6)$ & $1.5(0.9-2.4)$ & $0.9(0.7-1.1)$ \\
\hline \multicolumn{7}{|l|}{ CURB-65 } \\
\hline Cohort 1 & $39.8(28.6-50.9)$ & $45.3(33.4-57.3)$ & $44.6(32.6-56.6)$ & $40.5(29.4-51.6)$ & $0.7(0.5-1.0)$ & $1.3(1-1.8)$ \\
\hline Cohort 2 & $31.2(26-36.4)$ & $76.3(72.1-80.4)$ & $50.3(43.1-57.4)$ & $59.0(54.8-63.2)$ & $1.3(1.0-1.7)$ & $0.9(0.8-1)$ \\
\hline Cohort 3 & $18.9(7.4-30.3)$ & $83.8(77.6-89.9)$ & $28.6(12.2-45)$ & $75.0(68.2-81.8)$ & $1.2(0.6-2.3)$ & $1(0.8-1.1)$ \\
\hline \multicolumn{7}{|l|}{ CRB-65 } \\
\hline Cohort 1 & $14.5(6.3-22.6)$ & $72.0(61.2-82.8)$ & $36.4(18.4-54.3)$ & $43.2(34.1-52.3)$ & $0.5(0.3-1)$ & $1.2(1.0-1.4)$ \\
\hline Cohort 2 & $10.5(7.0-14)$ & $93.4(90.9-95.9)$ & $54.9(41.6-68.0)$ & $57.5(53.8-61.3)$ & $1.6(1-2.6)$ & $1(0.9-1.0)$ \\
\hline Cohort 3 & $7.6(0-15.6)$ & $92.2(87.7-96.8)$ & $25.0(0.7-49.3)$ & $74.4(67.9-80.8)$ & $1(0.3-2.9)$ & $1.0(0.9-1.1)$ \\
\hline
\end{tabular}


Table S3. Operative characteristics for mortality prediction in qSOFA (with cut-off point $\geq 2$ ), CURB 65 (with cut-off point $\geq 3$ ) and CRB 65 (with cut-off point $\geq 3$ ).

\begin{tabular}{|c|c|c|c|c|c|c|}
\hline & $\begin{array}{c}\text { Sensitivity } \\
(95 \% \text { CI })\end{array}$ & $\begin{array}{c}\text { Specificity } \\
(95 \% \text { CI })\end{array}$ & $\begin{array}{c}\text { PPV } \\
(95 \% \mathrm{CI})\end{array}$ & $\begin{array}{c}\text { NPV } \\
(95 \% \text { CI })\end{array}$ & $\mathrm{LR}+(\mathbf{9 5 \%} \mathrm{CI})$ & LR - $(95 \%$ CI $)$ \\
\hline \multicolumn{7}{|l|}{ qSOFA } \\
\hline Cohort 1 & $56.9(42.3-71.4)$ & $43.9(34.1-53.8)$ & $32.6(22.3-42.9)$ & $68.1(56.4-79.8)$ & $1.0(0.8-1.4)$ & $1(0.7-1.4)$ \\
\hline Cohort 2 & $39.1(30.2-47.9)$ & $73.6(70.0-77.1)$ & $23.5(17.6-29.4)$ & $85.3(82.2-88.4)$ & $1.5(1.2-1.9)$ & $0.8(0.7-1)$ \\
\hline Cohort 3 & $36.8(20.2-53.5)$ & $79.9(73.5-86.2)$ & $29.2(15.3-43.1)$ & $84.9(79.0-90.8)$ & $1.8(1.1-3.1)$ & $0.8(0.6-1.0)$ \\
\hline \multicolumn{7}{|l|}{ CURB-65 } \\
\hline Cohort 1 & $58.8(44.3-73.3)$ & $58.9(49.1-68.7)$ & $40.5(28.7-52.4)$ & $75.0(65.1-84.9)$ & $1.4(1.0-2)$ & $0.7(0.5-1.0)$ \\
\hline Cohort 2 & $45.3(36.3-54.3)$ & $76.8(73.4-80.2)$ & $28.9(22.3-35.4)$ & $87.1(84.2-90.0)$ & $2(1.5-2.3)$ & $0.7(0.6-0.8)$ \\
\hline Cohort 3 & $31.6(15.5-47.7)$ & $86.4(80.9-91.9)$ & $34.3(17.1-51.4)$ & $84.9(79.2-90.5)$ & $2.3(1.3-4.2)$ & $0.8(0.6-1)$ \\
\hline \multicolumn{7}{|l|}{ CRB-65 } \\
\hline Cohort 1 & $29.4(15.9-42.9)$ & $83.2(75.6-90.7)$ & $45.5(27-64)$ & $71.2(62.9-79.5)$ & $1.8(1-3.2)$ & $0.9(0.7-1.0)$ \\
\hline Cohort 2 & $14.1(7.7-20.5)$ & $92.9(90.9-95)$ & $29.0(16.9-41.1)$ & $83.9(81.1-86.7)$ & $2(1.1-3.3)$ & $0.9(0.7-1)$ \\
\hline Cohort 3 & $13.2(1.1-25.2)$ & $93.5(89.5-97.5)$ & $31.3(5.4-57.1)$ & $82.7(77.1-88.4)$ & $2.0(0.8-5.5)$ & $0.9(0.8-1.1)$ \\
\hline
\end{tabular}

\section{Figures}

\section{ICU with qSOFA (Cohort 1)}

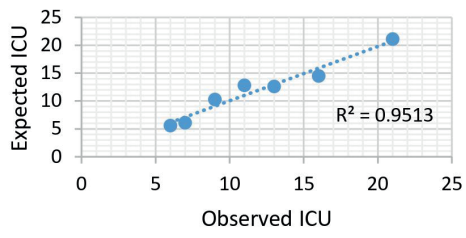

ICU with CURB (Cohort 1)

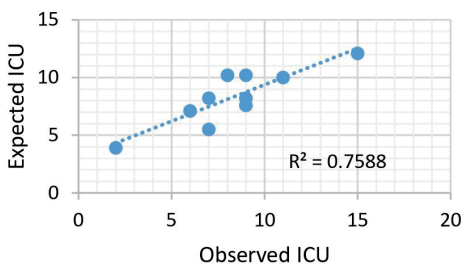

ICU with CRB (Cohort 1)

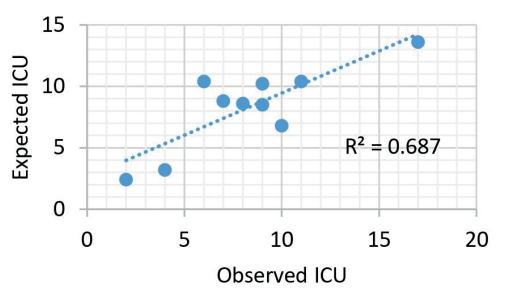

ICU with qSOFA (Cohort 2)

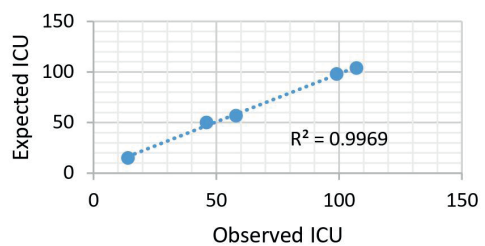

ICU with CURB (Cohort 2)

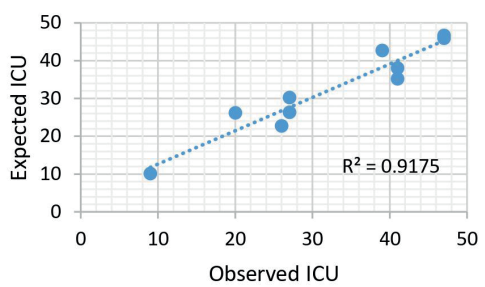

ICU with CRB (Cohort 1)

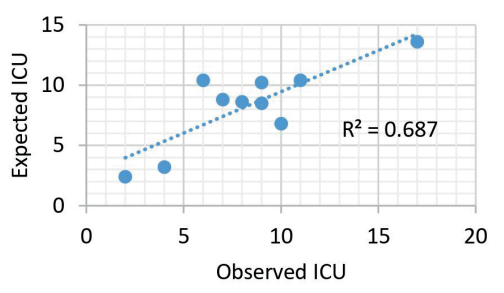

ICU with qSOFA (Cohort 3)

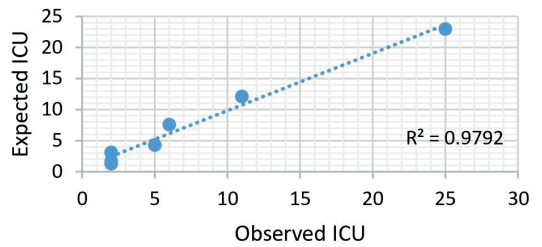

ICU with CURB (Cohort 3)

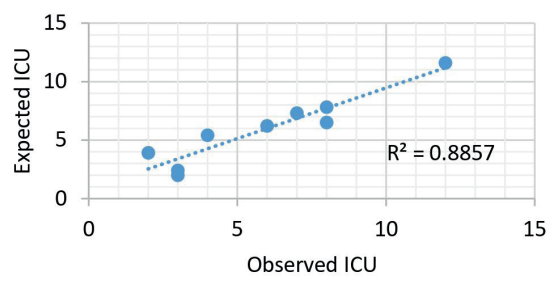

ICU with CRB (Cohort 2)

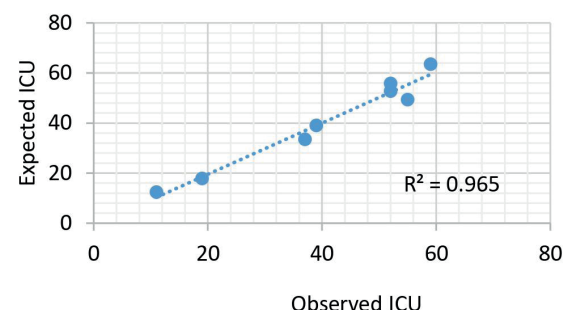

Figure S1. Calibration curves for ICU need prediction 
Mortality with qSOFA (Cohort 1)

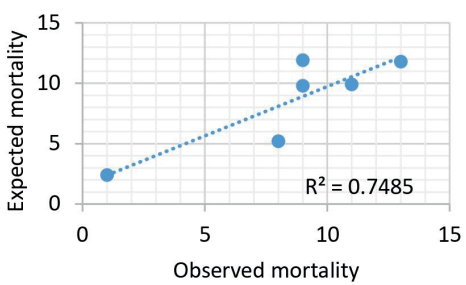

Mortality with CURB (Cohort 1)

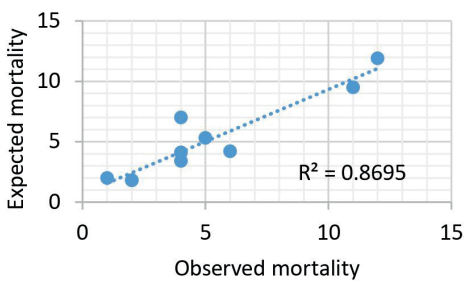

Mortality with CRB (Cohort 1)

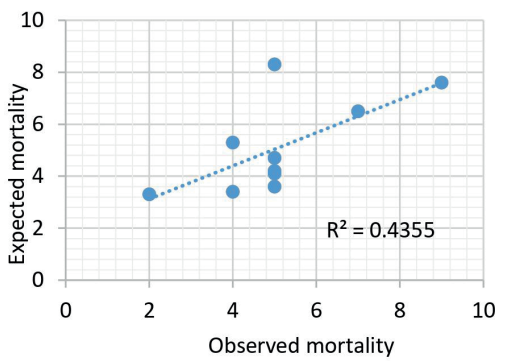

Mortality with qSOFA (Cohort 2)

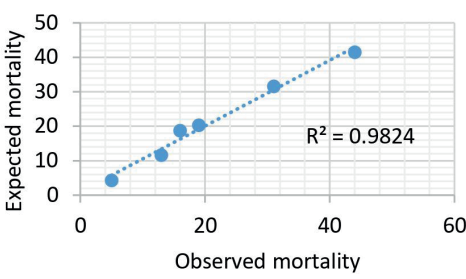

Mortality with CURB (Cohort 2)

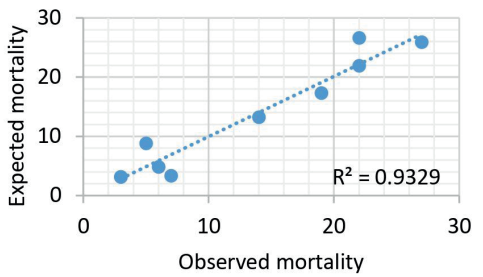

Mortality with CRB (Cohort 2)

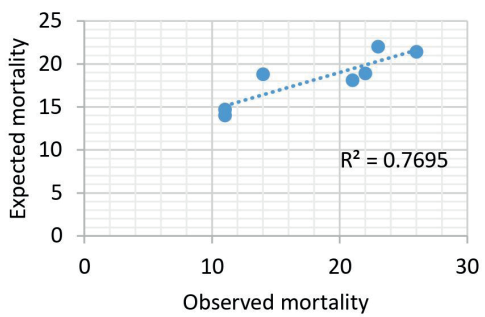

Mortality with qSOFA (Cohort 3)

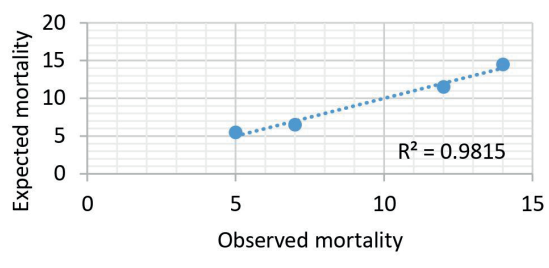

Mortality with CURB (Cohort 3)

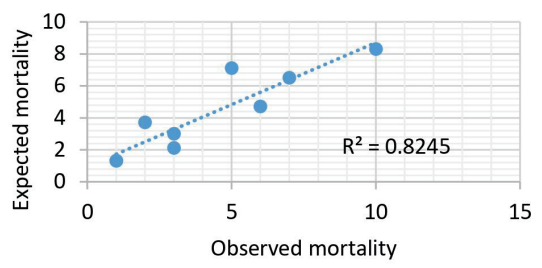

Mortality with CRB (Cohort 3)

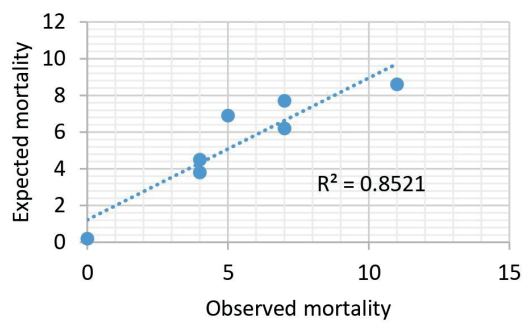

Figure S2. calibration curves for mortality prediction 